\title{
A Self Organizing Social Insect Model for Dynamic Frequency Allocation in Cellular Telephone Networks
}

\author{
Michael Lawlor, Tony White \\ School of Computer Science, Carleton University, Canada \\ +1 (613) 520-2600 x2208 \\ mike_lawlor@rogers.com, arpwhite@scs.carleton.ca
}

\begin{abstract}
This paper describes a mobile agent solution to the problem of frequency allocation encountered in cellular telephone networks. The mobile agents used follow a social insect behavioral model for division of labor and task allocation similar to that exhibited by swarms of ants, wasps and other social insects. Through this behavioral model, the "swarm" of agents self-organizes and displays an adaptive, swarm level intelligence that is transparent to each individual agent. Putting these agents to work in a simulated cellular telephone network, a simpler, distributed solution to frequency allocation was achieved.
\end{abstract}

\section{Categories and Subject Descriptors}

I.2.1 [Artificial Intelligence]: algorithms for intelligent allocation of frequencies in cellular networks.

\section{General Terms}

Algorithms, Experimentation.

\section{Keywords}

Swarm Intelligence, Mobile Agents, Cellular Telephone Network, Frequency Allocation

\section{INTRODUCTION}

A cellular network typically uses a fixed distribution of frequencies organized by cell in order to support voice calls. In order for a telephone call to be connected in such a network, its cell-phone must first request the allocation of an available frequency. The goal of this work was to reduce the number of calls unable to connect due to a lack of frequencies experienced by a cellular telephone network from that of a system with a fixed frequency distribution by dynamically reallocating frequencies to regions of high demand. The paper uses task specialization algorithms inspired by behaviour seen in social insect systems.

\section{The Mobile Agent Solution}

The mobile agents used in this paper are able to move about freely within the network from cell to cell. Every agent contains a 2dimensional array of threshold values associated with each cell in the network. These thresholds [2], [5] and [1] help agents

Copyright is held by the author/owner(s). $A A M A S$ '03, July 14-18, 2003, Melbourne, Australia. ACM 1-58113-683-8/03/0007.

determine whether they will respond to the demand for frequencies from the corresponding cell. If the intensity of a stimulus is at or above the threshold of a specific agent, then that agent will respond to the stimulus by changing to an "In Transit" state and moving towards the demanding cell. Once there, the agent switches to a "Working" state and performs its frequency allocation duties as outlined in the algorithm below. If the stimulus is below the agent's threshold, the agent may choose to ignore the stimulus and remain in its "Idle" state. Agents in the "idle" state wander randomly throughout the network until suitably stimulated. This random wandering is intended to mimic the aimless wandering of an un-stimulated ant in nature. Pseudocode for the algorithm employed by the mobile agents when allocating frequencies is shown below. This algorithm is executed once per tick while the agent is in the working state.

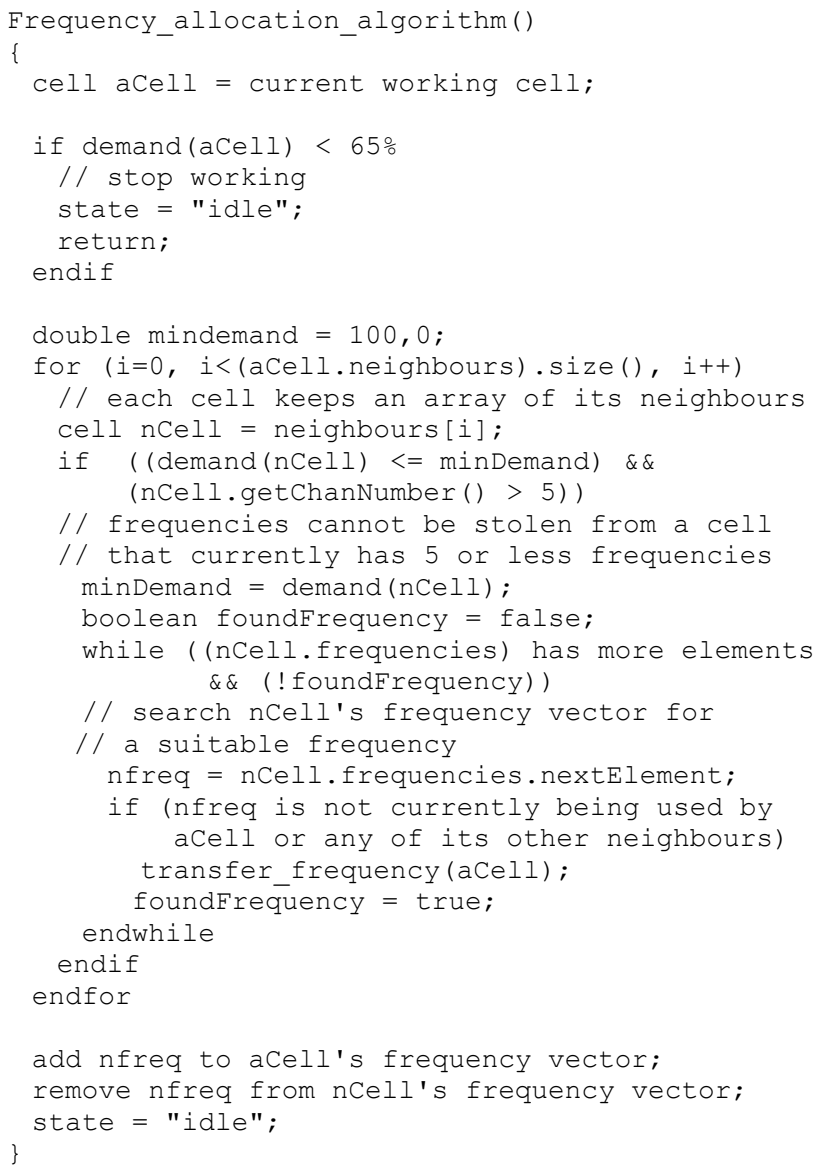


While the algorithm above does not allow for an agent to stop attempting to satisfy the demand for frequencies in a cell once it had started the search, adding the ability to prematurely terminate the search did not improve the overall results, although we hypothesize that it might provide some small improvement in extremely high network-wide frequency utilization scenarios.

The stimulation that a specific cell's demand exerts on an agent is determined by an equation that takes into account the agent's threshold, its current distance from the cell, and the actual amount of demand that the cell is experiencing (see Equation 1).

$P_{i, j}=\frac{S_{j}^{2}}{S_{j}^{2}+\alpha \theta_{a, j}^{2}} \frac{\beta d_{i, j}^{2}}{10}$

Equation 1

$\mathrm{S}_{\mathrm{j}} \quad$ the amount demand or stimulus of cell $j$

$\theta_{\mathrm{a}, \mathrm{j}} \quad$ the response threshold of agent $a$ to a demand from cell $j$.

$\mathrm{d}_{\mathrm{i}, \mathrm{j}} \quad$ is the distance between cell $i$ and cell $j$.

$\alpha, \beta \quad$ two positive coefficients that weight the influences of the threshold and the distance on the probability

In order to facilitate the division of labor, the mobile agents are capable of a simple form of learning [1], [2] and [5]. Initially each agent is given the same mean threshold value for every cell in the network. However, when an agent performs work at a given cell, the agent "learns" information about that cell and its neighbors. What this learning amounts to is a lowering of the agent's response threshold to future stimulation from this cell and its neighbors. Therefore, in future instances when cells of this region are in demand, the agent will have a higher probability of responding to their stimulation. Thus its can be said that the agent has specialized in this particular grid region. Conversely, the longer an agent goes without working in a specific cell, the greater the agent's threshold for that cell will become. It can therefore be said that an agent "forgets" cells that it has not recently worked in. This concept of division of labor is used in tandem with an adaptive task allocation system [5], [2] to manage the agents in such a way that the global demand in the network is kept as low as possible (see Equations 2, 3, and 4).

$\theta_{\mathrm{a}, \mathrm{j}}=\theta_{\mathrm{a}, \mathrm{j}}-\xi_{0}$

Equation 2

$n(j) \quad$ is the set containing all of $j$ 's neighbouring cells.

$\xi_{0} \quad$ is the learning coefficient for cell $\mathrm{j}$.

$\theta_{\mathrm{a}, \mathrm{n}(\mathrm{j})}=\theta_{\mathrm{a}, \mathrm{n}(\mathrm{j})}-\xi_{1}$

Equation 3

for all $n(j)$

$\xi_{1} \quad$ is the learning coefficient for cell j's neighbors.

$\theta_{\mathrm{a}, \mathrm{k}}=\theta_{\mathrm{a}, \mathrm{k}}+\varphi$

for all $\mathrm{k}$ not equal to $\mathrm{j}$,

and not contained in $\mathrm{n}(\mathrm{j})$

$\varphi \quad$ is the forgetting coefficient for all other cells in the grid.

The mobile agent software was composed solely of the mobile agents. Each mobile agent kept track of its position in the grid, its response thresholds for every cell of the grid, and it's learning and forgetting coefficients (see Equations 2, 3, and 4). The initial values of $\alpha, \beta, \theta_{\mathrm{aj}}, \xi_{0}, \xi_{1}$ and $\varphi$ were $0.5,50,50,15,7,0.5$ respectively. Mobile agents were all capable of movement at 1 cell/second when in transit between cells. These values were determined empirically and found to provide reasonable results with the call generation and holding times used in the experiments.

\section{DISCUSSION AND CONCLUSIONS}

Based on the experimental results, and their subsequent analysis, the solution proposed in this paper can be considered a promising solution for the dynamic frequency assignment problem. In the very worst test case results, the mobile agent method performed the same or just slightly worse than the fixed frequency configuration method. In the majority of cases where more reasonable values were used for the call generation rate, there was an observed number of mobile agents that when added to the network improved its ability to allocate frequencies and reduced blocked calls. In some cases the improvement was quite large (reducing blocked calls by $75 \%$ ), while not so in others (less than $10 \%)$. However, where an improvement was detected, the percentage of blocked calls was reduced to a fraction of its original level (typically 15-20\%).

The results reported are promising but not conclusive. Clearly, comparison with other dynamic frequency allocation algorithms is warranted [3], [4]. This paper has demonstrated that the use of mobile agents with division of labor and adaptive task allocation can be applied to situations once reserved for more complex centralized management systems. A solution similar to the one demonstrated here might one day be considered by a cellular provider or implemented in an actual cellular network. As these cellular networks become larger and more complex, the need for them to self-organize will only increase. Using inspiration taken from the behavior of insect societies - systems finely tuned by millions of years of evolution - a more distributed, more organic solution can be applied to cellular networks which have themselves become complex organisms.

\section{REFERENCES}

[1] Bonabeau, E., Dorigo, M., Theraulaz, G., Inspiration for optimization from social insect behaviour. Nature, 406:3942, July 2000.

[2] Bonabeau E., Sobkowski A., Theraulaz G., and Deneubourg J.L., Adaptive task allocation inspired by a model of division of labor in social insects. In D. Lundh and B. Olsson, editors, BioComputation and Emergent Computing, pages 36-45, World Scientific, 1997.

[3] Chao C. L. I. and Chao P. H., Local packing-distributed dynamic channel allocation at cellular base station. GLOBECOM'93, 1993.

[4] Chuang J. C.-I., Performance issues and algorithms for dynamic channel assignment. IEEE Journal on Selected Areas in Communications, 11(6):955-963, 1993.

[5] Theraulaz G., Bonabeau E. and Deneubourg J.L., Response threshold reinforcement and division of labour in insect societies. Proceedings of the Royal Society of London B, 265(1393):327-335, February 1998. 